\title{
Exudative Glomerulonephritis
}

National Cancer Institute

\section{Source}

National Cancer Institute. Exudative Glomerulonephritis. NCI Thesaurus. Code C35706.

Inflammation of the glomeruli with infiltration by polymorphonuclear leukocytes. 